\title{
The Herbivore-Induced Plant Volatile Methyl Salicylate Negatively Affects Attraction of the Parasitoid Diadegma semiclausum
}

\author{
Tjeerd A. L. Snoeren • Roland Mumm • \\ Erik H. Poelman • Yue Yang • Eran Pichersky • \\ Marcel Dicke
}

Received: 5 January 2010 /Revised: 6 April 2010/Accepted: 8 April 2010/Published online: 21 April 2010

(C) The Author(s) 2010. This article is published with open access at Springerlink.com

\begin{abstract}
The indirect defense mechanisms of plants comprise the production of herbivore-induced plant volatiles that can attract natural enemies of plant attackers. One of the often emitted compounds after herbivory is methyl salicylate (MeSA). Here, we studied the importance of this caterpillar-induced compound in the attraction of the parasitoid wasp Diadegma semiclausum by using a mutant Arabidopsis line. Pieris rapae infested AtBSMT1-KO mutant Arabidopsis plants, compromised in the biosynthesis of MeSA, were more attractive to parasitoids than infested wild-type plants. This suggests that the presence of MeSA has negative effects on parasitoid host-finding behavior when exposed to wild-type production of herbivoreinduced Arabidopsis volatiles. Furthermore, in line with this,
\end{abstract}

Electronic supplementary material The online version of this article (doi:10.1007/s10886-010-9787-1) contains supplementary material, which is available to authorized users.

T. A. L. Snoeren $(\bowtie) \cdot$ R. Mumm $\cdot$ E. H. Poelman $\cdot$ M. Dicke Laboratory of Entomology, Wageningen University, P.O. Box 8031, $6700 \mathrm{EH}$ Wageningen, the Netherlands e-mail: tjeerd.snoeren@wur.nl

\section{Y. Yang $\cdot$ E. Pichersky}

Department of Molecular, Cellular and Developmental Biology, University of Michigan,

Ann Arbor, MI 48109, USA

Present Address:

R. Mumm

Plant Research International B.V.,

P.O. Box 619,

6700 AP Wageningen, the Netherlands

Present Address:

Y. Yang

Department of Plant Biology, Michigan State University,

East Lansing, MI 48824-1312, USA we recorded a positive correlation between MeSA dose and repellence of $D$. semiclausum when supplementing the headspace of caterpillar-infested AtBSMT1-KO plants with synthetic MeSA.

Key Words Herbivore-induced plant volatile.

SABATH methyl transferase $\cdot B S M T 1 \cdot$ Methyl salicylate .

Parasitoid host-location behaviour · Herbivory .

Volatile emission

\section{Introduction}

Plants effectively combat herbivorous insects through direct and indirect defenses (Kessler and Baldwin 2002). Direct defense comprises the production and storage of metabolites that negatively influence herbivore performance (Wittstock and Gershenzon 2002). In contrast, indirect defense encompasses the production of metabolites that benefit the natural enemies of herbivores (Dicke et al. 1999; Wäckers et al. 2001; Halitschke et al. 2008; Kost and Heil 2008). Natural enemies such as parasitoids use herbivoreinduced plant volatiles (HIPVs) to locate their herbivore host and thereby indirectly aid plants in their combat against herbivorous insects. HIPVs are complex blends of compounds and mainly comprise green leaf volatiles (GLV) (C6 aldehydes, alcohols, and derivatives), terpenoids, and phenolics (Paré and Tumlinson 1997; Dicke 1999b). Green leaf volatiles originate from linolenic and linoleic acid, which are released particularly when cells are damaged (Bate and Rothstein 1998). Terpenoids are synthesized via the mevalonic acid (MVA) or methylerythritol phosphate (MEP) pathway (Dudareva et al. 2006; Gershenzon and Dudareva 2007). Finally, aromatic compounds, such as 
methyl salicylate (MeSA) and indole, are formed via the shikimic acid pathway (Paré and Tumlinson 1997). The induced volatile production is orchestrated by at least three main signal-transduction pathways: the jasmonic acid (JA), salicylic acid (SA), and ethylene (ET) pathways (Dicke and Van Poecke 2002; Kessler and Baldwin 2002). These pathways can be induced differentially by different herbivore species (Heidel and Baldwin 2004; De Vos et al. 2005; Schmidt et al. 2005), leading to the emission of a volatile blend that is specific for an herbivore species (Vet and Dicke 1992; Ozawa et al. 2000; Walling 2000; Leitner et al. 2005). These herbivore-specific volatile blends can provide foraging natural enemies of herbivores, such as predators and parasitoid wasps, with detectable and reliable information to locate their prey or host respectively (Dicke et al. 1990; Turlings et al. 1991; Vet and Dicke 1992; Du et al. 1998; Dicke 1999a).

Variation in attraction of carnivorous arthropods towards host or non-host infested plants is ascribed mainly to the presence and relative abundance of attractive compounds within the HIPV blend (D'Alessandro et al. 2009). An intriguing question is which components of the complex HIPV blend affect parasitoid attraction most. This will be different for different parasitoid species. Up to now, several approaches have been applied to study the relative importance of certain HIPVs in the attraction of carnivorous arthropods, e.g., by offering synthetic compounds alone or in mixtures, inducing certain subsets of the HIPV blend with elicitors, or manipulating signal-transduction or biosynthetic pathways through a molecular genetic approach (for review see D'Alessandro and Turlings 2006; Snoeren et al. 2007; Schroeder and Hilker 2008). One HIPV component for which biological relevance for carnivorous arthropod attraction has been addressed is methyl salicylate (MeSA) (De Boer and Dicke 2004; De Boer et al. 2004; James and Price 2004; Zhu and Park 2005; Ishiwari et al. 2007). This methyl ester of the plant hormone salicylic acid (SA) has been reported in the HIPV blends emitted by several plant species, e.g., lima bean (Dicke et al. 1990), tomato (Ament et al. 2004), cabbage (Geervliet 1997; Poelman et al. 2009), and Arabidopsis (Van Poecke et al. 2001; Chen et al. 2003; Snoeren et al. 2009). Its role in the attraction of carnivorous arthropods has been addressed by investigating the response towards synthetic MeSA in field (James and Price 2004; Zhu and Park 2005) and in laboratory studies (Dicke et al. 1990; De Boer and Dicke 2004; De Boer et al. 2004; Ishiwari et al. 2007). However, so far, no studies have addressed the effects of the absence of MeSA from an otherwise complete HIPV blend on the behavior of carnivorous arthropods.

In this study, we focused on the function of MeSA within the HIPV blend through a molecular ecological approach that involves the elimination of MeSA. MeSA is synthe- sized by SA carboxyl methyltransferase (SAMT), a member of the SABATH methyl transferase family, to which jasmonic acid, indole-acetic acid and cinnamic $/ p$ coumaric acid methyltransferases also belong (Seo et al. 2001; Chen et al. 2003; Zubieta et al. 2003; Kapteyn et al. 2007). Related enzymes that methylate benzoic acid (BA) to give MeBA also have been reported (Murfitt et al. 2000). Some SABATH enzymes can methylate both SA and BA with roughly equal efficiencies, and have been designated as Benzoic Acid and Salicylic Acid Methyl Transferases (BSMTs). One such example is the Arabidopsis thaliana gene designated BSMT1 (Chen et al. 2003).

While SAMT or BSMT enzymes have been identified in a number of plant species, including fairy fans (Clarkia breweri), snapdragon (Antirrhinum majus), petunia (Petunia hybrida), Arabidopsis, and jasmine (Stephanotis floribunda), we selected Arabidopsis for further work on the physiological significance of MeSA emission because of the availability of molecular genetic tools and because this species is a valuable stepping stone towards other brassicaceous plants for studying the role of HIPVs in plant-insect interactions (Snoeren et al. 2007). We addressed the role of MeSA in the foraging behavior of the parasitoid wasp Diadegma semiclausum Hellén (Hymenoptera, Ichneumonidae) that attacks caterpillars feeding on brassicaceous plants including Arabidopsis. Caterpillar feeding is known to induce MeSA in Arabidopsis (Van Poecke et al. 2001; Chen et al. 2003; Snoeren et al. 2009). We used wild-type Arabidopsis plants and a knock-out mutant that does not have a functional BSMT and, thus, no MeSA biosynthesis. We addressed the effects of the mutation on parasitoid- and herbivore behavior, and on headspace composition.

\section{Methods and Materials}

Plants and Insect Material An Arabidopsis line with an insertion in the AtBSMT1 gene was obtained from the Torrey Mesa Institute collection, and the position of the insertion was verified by sequencing (Fig. 1, see also supporting information Fig. S1). Arabidopsis seeds ( $A$. thaliana wild-type Columbia (Col-0) and AtBSMT1-KO in a Col-0 background) were germinated in sandy Arabidopsis soil (Lentse potgrond BV, Lent, Netherlands), and cultivated in a growth chamber at $21 \pm 2^{\circ} \mathrm{C}, 50$ to $60 \%$ relative humidity (RH), and 8:16 hr, L:D photoperiod with 80 to $110 \mu \mathrm{mol} \mathrm{m}{ }^{-2} \mathrm{sec}^{-1}$ PPFD. The soil was heated to $90^{\circ} \mathrm{C}$ for at least $2 \mathrm{hr}$ prior to sowing of the plants. Two-wk-old seedlings were transferred to plastic containers $(5 \mathrm{~cm}$ diam $)$ filled with the same soil type. Plants were watered twice a week. To prevent infestation by root-feeding sciarid flies, the soil was treated weekly with the entomopathogenic nematode Steinernema feltiae (Koppert Biological Systems, 


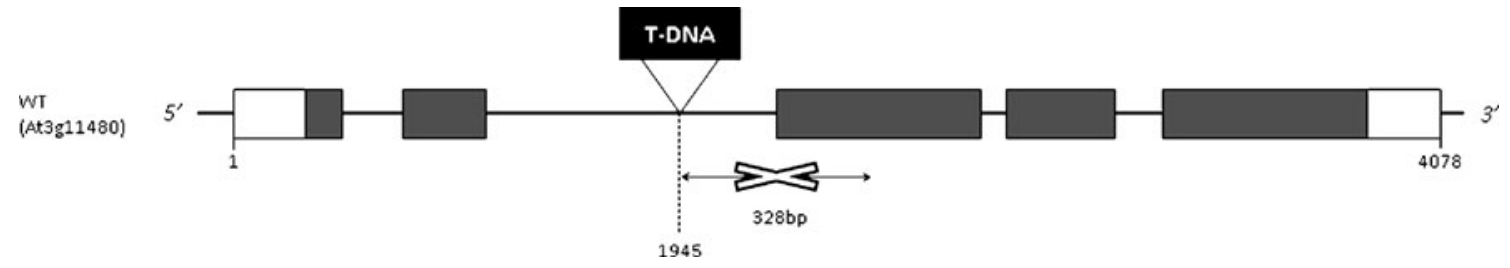

Fig. 1 Illustration of the AtBSMT T-DNA mutant (GARLIC 776 B10 Line) from The Torrey Mesa Institute's T-DNA insertion mutant collection. The exons are indicated as grey boxes and nucleotide numbers are given below. The position of the approximately 200-nt T-DNA insertion, as well as a deletion of 328-nt extending downstream from the right border of the T-DNA element and including the end part of intron 2 and the beginning part of exon 3 also are shown. Both insertion and deletion were determined by

Berkel en Rodenrijs, the Netherlands). Fully grown vegetative plants were used for the experiments, i.e., at 68 wk after sowing.

The small cabbage white butterfly, Pieris rapae L. (Lepidoptera, Pieridae), was reared on Brussels sprouts plants (Brassica oleracea var. gemmifera, cv Cyrus) in a growth chamber $\left(16: 8 \mathrm{hr}, \mathrm{L}: \mathrm{D} ; 20 \pm 2^{\circ} \mathrm{C}\right.$ and $\left.70 \% \mathrm{RH}\right)$ as described in detail in Fatouros et al. (2005).

The parasitoid wasp D. semiclausum was reared on Plutella xylostella on Brussels sprouts in a climatized room (16:8 hr, L:D; $20 \pm 2^{\circ} \mathrm{C}$ and $\left.70 \% \mathrm{RH}\right)$. Emerging wasps were provided ad libitum with water and honey, and are referred to as 'naïve' wasps, as they had received neither exposure to plant material, nor obtained an oviposition experience. This parasitoid is known to be attracted to the volatiles emitted by $P$. rapae-infested Arabidopsis Col-0 plants (Loivamäki et al. 2008).

Plant Treatments Plants were infested by equally distributing 20 first-instar P. rapae larvae per plant over the fully expanded leaves. Uninfested plants that otherwise received a treatment similar to the infested plants were used as controls. In all experiments, plants from both genotypes that required an infestation were treated $24 \mathrm{hr}$ before the experiments and kept in a climate room $\left(21 \pm 2^{\circ} \mathrm{C}, 50-60 \%\right.$ $\mathrm{RH}$; 8:16 hr, L:D photoperiod, and $80-110 \mu \mathrm{mol} \mathrm{m}^{-2} \mathrm{sec}^{-1}$ PPFD).

Y-tube Olfactometer Behavioral Assays The effects of HIPVs from wild-type Arabidopsis Col-0 and transgenic AtBSMT1-KO plants on parasitoid behavior were tested in a closed-system Y-tube olfactometer as described by Bukovinszky et al. (2005). In short, filtered air was led through activated charcoal and split into two air streams $\left(4 \mathrm{~L} \mathrm{~min}^{-1}\right)$ that were led through five-liter glass vessels containing the odor sources consisting on each experimental day of 4 plants each with the feeding caterpillars. The olfactometer was illuminated with 4 high-frequency fluorescent tubes (Philips 840, $36 \mathrm{~W}$ ) from above at an intensity sequencing. Furthermore, we determined that transcripts from the mutant gene were still produced in the plant, and the processed mRNA had a similar size to that of the wild type (WT) mRNA. However, when the mutant transcript was amplified by RT-PCR and sequenced, it was seen that in the absence of the original $3^{\prime}$ end of intron 2, a new $3^{\prime}$ splice site was used within the remains of exon 3. This led to a protein that is much shorter and does not have SAMT activity (See also Fig. S1)

of $60 \pm 5 \mu \mathrm{mol} \mathrm{m} \mathrm{mec}^{-1}$ PPFD. All experiments were conducted in a climatized room $\left(20 \pm 2^{\circ} \mathrm{C}\right)$.

Naïve, 3-7-d-old female D. semiclausum were transferred individually from the cage into the Y-tube olfactometer on a plant leaf; this was done by using alternately a Col-0 or AtBSMT1-KO leaf, that had been infested previously by $P$. rapae and from which the caterpillars and their products had been removed carefully. This procedure increases the general behavioral response of the parasitoids to plant cues but does not induce a shift of preference (Kaiser and Carde 1992; Bleeker et al. 2006; Smid 2006). Upon release in the olfactometer, parasitoid behavior and parasitoid choice for one of the two odor sources was observed and scored as described in detail by Bukovinszky et al. (2005). Parasitoids that did not explore one of the two arms of the olfactometer within $5 \mathrm{~min}$ or that did not make a final choice within 10 min after release were considered as non-responding individuals, and were excluded from preference analysis. After every 5 parasitoids tested, the odor sources were interchanged to compensate for any unforeseen asymmetry in the set-up.

Experiment 1. Effect of AtBSMT Knock-out on Parasitoid Attraction to Caterpillar-infested Plants To assess the role of MeSA as a cue for parasitoids in planta, we compared parasitoid behavior in response to an HIPV blend that lacked MeSA vs. a complete HIPV blend that included MeSA. Caterpillar-infested mutant AtBSMT1-KO and wildtype Col-0 plants were offered as odor sources in the olfactometer to D. semiclausum.

Experiment 2. Supplementing Headspace of Caterpillarinfested AtBSMT1-KO Plants with Synthetic MeSA To investigate further the role of MeSA, we supplemented the HIPV blend of AtBSMT1-KO plants by adding synthetic MeSA (Merck, 99\% pure). The supplemented HIPV blend from these AtBSMT1-KO plants was tested against the emitted HIPV blend from AtBSMT1-KO or Col-0 plants. To determine if parasitoid behavior to MeSA was dose 
dependent, different doses of MeSA $(0.2 \mu \mathrm{g}, 2 \mu \mathrm{g}, 20 \mu \mathrm{g}$, $200 \mu \mathrm{g}$ ) were added downwind to the earlier infested AtBSMT1-KO plants. MeSA was diluted in n-hexane (Sigma-Aldrich, 95\%). In all experiments, $0.1 \mathrm{ml}$ of the MeSA solution was applied on filter paper $\left(15 \mathrm{~cm}^{2}\right)$ and positioned in the last section of the olfactometer arm. A piece of filter paper with $0.1 \mathrm{ml}$ hexane was placed at a similar position in the other arm as a control. The solvent was allowed to evaporate for 30 to $60 \mathrm{sec}$, after which a parasitoid was introduced into the olfactometer. New filter papers with MeSA or hexane were used for each parasitoid tested in the olfactometer.

Caterpillar-feeding Areas of consumed leaf-tissue were assessed for the caterpillar-infested Col-0 and AtBSMT1$K O$ plants that were used in experiment 1 . Immediately after finishing an olfactometer bioassay, individual leaves were taped on paper and scanned with a Hewlett-Packard scan jet 3570c. Original leaf shapes were reconstructed by using Paint.NET v3.30, Microsoft Corporation. Quantification of consumed leaf-tissue area was performed using Winfolia pro 2006a, Regent instruments (Québec, Canada).

Headspace Collection And Volatile Analysis Dynamic headspace sampling was carried out in a climate room $\left(20 \pm 2^{\circ} \mathrm{C}, 70 \% \mathrm{RH} ; 8: 16 \mathrm{hr}\right.$, L:D photoperiod and 90 to $110 \mu \mathrm{mol}$ photons $\left.\mathrm{m}^{-2} \mathrm{sec}^{-1} \mathrm{PPFD}\right)$. Twenty-four $\mathrm{h}$ before sampling, the pots were removed, roots and soil were carefully wrapped in aluminum foil, and 4 plants were placed together in a $2.5 \mathrm{~L}$ glass jar. The glass jars then were covered with insect-proof gauze. Just before trapping, the gauze was removed and the jars were closed with a Viton-lined glass lid having an inlet and outlet. Inlet air was filtered by passing through tubes filled with $200 \mathrm{mg}$ Tenax TA (20/35 mesh; Grace-Alltech, Deerfield, MI, USA). Air was sucked out of the jar with $100 \mathrm{ml} \mathrm{min}^{-1}$ by passing through a tube filled with $200 \mathrm{mg}$ Tenax TA. Headspace volatiles from different treatments were collected for a period of $3.5 \mathrm{hr}$. Fresh weights of all rosettes were determined immediately after the experiments. On each experimental day, headspace samples for two or three replicates of each treatment were collected simultaneously.

Headspace samples were analyzed with a Thermo TraceGC Ultra (Thermo Fisher Scientific, Waltham, MA, USA) connected to a Thermo TraceDSQ (Thermo Fisher Scientific, Waltham, MA, USA) quadrupole mass spectrometer. Before desorption of the volatiles, the Tenax cartridges were dry-purged with helium at $30 \mathrm{ml} \mathrm{min}^{-1}$ for $20 \mathrm{~min}$ at ambient temperature to remove moisture. Samples were desorbed from the cartridges by using a thermal desorption system at $250^{\circ} \mathrm{C}$ for $3 \mathrm{~min}$ (Model Ultra Markes Llantrisant, UK) with a helium flow at $30 \mathrm{ml} \mathrm{min}^{-1}$. Analytes were focused at $0^{\circ} \mathrm{C}$ on an electronically-cooled sorbent trap filled with Tenax and Carbograph (Unity ${ }^{\mathrm{TM}}$, Markes International LTD, Llantrisant, UK) and were then transferred in splitless mode to the analytical column (Rtx-5 ms, $30 \mathrm{~m}, 0.25 \mathrm{~mm}$ i.d., $1.0 \mu \mathrm{m}$ film thickness, Restek, Bellefonte, PA, USA) by rapid heating of the cold trap to $250^{\circ} \mathrm{C}$. The $\mathrm{GC}$ was held at an initial temperature of $40^{\circ} \mathrm{C}$ for $3.5 \mathrm{~min}$, followed by a linear thermal gradient of $10^{\circ} \mathrm{C} \min ^{-1}$ to $280^{\circ} \mathrm{C}$, and held for $2.5 \mathrm{~min}$ with a column flow of $1 \mathrm{ml} \mathrm{min}$. The column effluent was ionized by electron impact ionization at $70 \mathrm{eV}$. Mass spectra were acquired by scanning from $45-400 \mathrm{~m} / \mathrm{z}$ with a scan rate of 3 scans $\mathrm{sec}^{-1}$.

Compounds were identified by using the deconvolution software AMDIS (version 2.64, NIST, USA) in combination with NIST 98 and Wiley 7th edition spectral libraries and by comparing their retention indices with those from the literature (Adams 1995). For quantification, characteristic quantifier ions were selected for each compound of interest. MetAlign software (PRI-Rikilt, Wageningen, the Netherlands) was used to remove baseline noise, to align the peaks of all chromatograms of the samples, and to integrate peak areas of quantifier ions. The peak areas of all compounds were corrected for the fresh weight of the leaf rosettes.

\section{Statistical Analysis}

Y-tube Olfactometer Behavioral Assays Parasitoid preference for infested AtBSMT1-KO vs. infested Col-0 plants (i.e., experiment 1 ) were analyzed statistically by using a Chisquare test, with the null-hypothesis that parasitoids did not have a preference for any of the two odor sources. Second, we analyzed whether the parasitoids exhibited a MeSAdose-dependent response when the HIPV blend from AtBSMT1-KO plants was supplemented with synthetic MeSA (i.e., experiment 2). We constructed an overall generalized linear model (GLM) including MeSA dose as a covariate, the tested genotype combination as a fixed factor, and the interaction of the terms. As the null-hypothesis, we defined that addition of MeSA did not affect attraction of parasitoids. Total number of parasitoids that preferred infested AtBSMT1-KO plants with the supplemented MeSA over the control (infested Col-0 or AtBSMT1-KO) plants was taken as response variate. Total numbers of parasitoids that made a choice per day were used as the binomial total, which were fixed on 10 except for a single case where 9 wasps responded, and we used a logit-link function. We used a Chi-square test, with the aforementioned nullhypothesis, to test for a significant preference of each tested MeSA dose per genotype combination. Parasitoids that did not make a choice were not included in the test.

Third, we investigated the effects of MeSA dose on the proportion of parasitoids that made a choice in the olfactometer experiments. We used the same overall GLM 
model as described above, only now with number of nonresponding parasitoids as the response variate (SPSS 15.0, Chicago, IL, USA).

A Mann-Whitney- $U$ test was used to test whether the consumed leaf area of Col-0 and AtBSMT1-KO plants (experiment 1) was different (SPSS 15.0, Chicago, IL, USA).

Headspace Collection The fresh weight of the corrected peak area for a volatile compound quantifier ion was $\log 10$ transformed, and for each HIPV, the following mixed model was used to screen for HIPV compound differentiation per genotype: $\log 10\left(\mathrm{~V}_{\mathrm{ijk}}\right) \sim \mathrm{G}_{\mathrm{i}}+\mathrm{T}_{\mathrm{j}}+\mathrm{G}: \mathrm{T}_{\mathrm{ij}}+\mathrm{R}_{\mathrm{k}}+\varepsilon_{\mathrm{ijk}}$, where $\mathrm{V}=$ area of quantifier ions per gram fresh weight; $\mathrm{G}=$ genotype; $\mathrm{T}=$ treatment; $\mathrm{R}=$ replicate; $\varepsilon=$ residual; $\mathrm{i}=1,2$; $\mathrm{j}=1,2$; and $\mathrm{k}=1, \ldots, 5$. Both $\mathrm{G}$ and $\mathrm{T}$ were used as fixed effects and $\mathrm{R}$ as a random effect. Subsequently, two-tailed $t$ tests followed by a Benjamini and Hochberg false discovery rate (BH-FDR) multiple comparison correction were conducted per compound for the genotypes [significance: $\mathrm{q}<0.05$; Benjamini and Hochberg 1995].

\section{Results}

Headspace Volatile Analysis To evaluate the effects of the BSMT knock-out mutation, we analyzed the headspace of uninfested and $P$. rapae-infested plants of wild-type Col-0 and AtBSMT1-KO mutant plants. For the analysis, we selected compounds in the HIPV blend that are known to influence the behavior of carnivorous arthropods, e.g., methyl salicylate, hydrocarbon alcohols, and terpenoids, and compounds predicted to be affected by the KO mutation, i.e. methyl salicylate and methyl benzoate (MeBA) (Fig. 2) (Turlings and Fritzsche 1999; Dicke et al. 1990; Chen et al. 2003; De Boer et al. 2004; Shimoda et al. 2005).

Infested Col-0 and AtBSMT1-KO plants differed significantly in the emission of MeSA, ethyl salicylate (EtSA) and MeBA $(q<0.001)$; these compounds were induced in Col-0 but not in AtBSMT1-KO. The Y-axis represents a ${ }^{10} \log$ scale. Thus, the headspace analysis for infested AtBSMT1-KO and infested Col-0 showed that the production of MeBA (No. 10), MeSA (No. 12), and ethyl salicylate (EtSA, No. 13) was hampered. These compounds are emitted from AtBSMT1-KO at 14, 59, and 17 times lower emission rates, respectively (Fig. 2).

Several other compounds-1-pentanol, linalool, indole, $(E, E)$ - $\alpha$-farnesene, and $(E, E)-4,8,12$-trimethyltrideca1,3,7,11-tetraene (TMTT) - similarly were induced in infested Col-0 and AtBSMT1-KO plants when compared to uninfested plants. The green leaf volatile $(Z)-3$-hexen-1ol was induced significantly in only the mutant $(q=0.005)$ but not in the wild-type. Uninfested Col-0 and AtBSMT1$K O$ plants did not differ in the emission of volatiles, except for
TMTT, which was emitted in somewhat larger amounts by uninfested Col-0 plants than by uninfested AtBSMT1-KO plants $(q=0.011)$. Finally, uninfested Col-0 plants emitted more 1-octen-3-ol and 1-nonanol than infested plants (Fig. 2).

\section{Y-Tube Olfactometer Behavioral Assays}

Experiment 1. Effect of AtBSMT Knock-out on Parasitoid Attraction to Caterpillar-infested Plants Diadegma semiclausum females are attracted to the headspace of $P$. rapaeinfested Arabidopsis Col-0 plants (Loivamäki et al. 2008) in which MeSA is an induced volatile compound (Van Poecke et al. 2001). To investigate whether changes in this headspace, as a result of a knock-out mutation in the AtBSMT1 gene, affected parasitoid attraction, we investigated the behavioral responses of $D$. semiclausum towards plant volatiles induced by $P$. rapae herbivory in a Y-tube olfactometer. Females of $D$. semiclausum preferred the volatiles emitted by herbivore-infested AtBSMT1-KO plants over those emitted by infested Col- 0 plants $(P<0.05$; Fig. 3a).

The amount of leaf-tissue consumed by the caterpillars did not differ between Col-0 and AtBSMT1-KO plants (mean \pm SE: Col-0 10.41 $\pm 0.91 \mathrm{~mm}^{2}$, AtBSMT1-KO 10.88 \pm $0.80 \mathrm{~mm}^{2}$; Mann Whitney $U$ test: $U=259.00 ; P=0.90, N=$ 23). Thus, the difference in attraction cannot be explained by a difference in the amount of feeding by the caterpillars.

Experiment 2. Supplementing Headspace of Caterpillarinfested AtBSMT1-KO Plants with Synthetic MeSA To assess whether the absence of MeSA in the HIPV blend can explain the effect on parasitoid behavior, the volatile blend of infested AtBSMT1-KO plants was supplemented by adding synthetic MeSA downwind of the plant. Different MeSA doses were used to test for dosedependent effects of MeSA presence. The addition of MeSA eliminated the preference for the knock-out plants, as seen in experiment 1, and with increasing MeSA dose this effect was stronger. Parasitoid preference was not influenced by the different genotype combinations, but solely by the MeSA dose used (GLM: genotype combination $P=0.167$, MeSA dose $P<0.001, R^{2}=0.28$ ). Analysis of parasitoid choices for each tested MeSA dose within a tested genotype combination demonstrated that parasitoid preferences slightly varied among the MeSA doses used. Yet, for both genotype combinations tested, AtBSMT1-KO HIPV complementation with $0.2 \mu \mathrm{g}$ MeSA did not result in discrimination between the two odor sources and a complementation with $200 \mu \mathrm{g}$ MeSA resulted in significant preference for the odor without supplemented MeSA (Fig. 3b, c). When parasitoids were offered a choice between volatiles from infested AtBSMT1-KO plants supplemented with 2 or $20 \mu \mathrm{g}$ MeSA vs. infested Col-0 HIPVs, 
Fig. 2 Emission of volatile compounds of Col-0 wild-type and AtBSMT-KO Arabidopsis plants, either uninfested or infested with 20 Pieris rapae caterpillars, expressed as peak area (arbitrary units; mean $\pm \mathrm{SE}$; $N=5$ ) for the identifying ion per g FW. Compounds: 1=1-pentanol $(\mathrm{m} / \mathrm{z} 70) ; 2=(Z)-2$-penten-1ol $(m / z 57) ; 3=(Z)-3$-hexen-1-ol $(\mathrm{m} / \mathrm{z}$ 67); 4= $\alpha$-pinene $(\mathrm{m} / \mathrm{z}$ 93); $5=1$-octen-3-ol $(\mathrm{m} / \mathrm{z} 57) ; 6=\beta$ myrcene ( $\mathrm{m} / \mathrm{z}$ 93); $7=(Z)-3$ hexen-1-ol acetate $(\mathrm{m} / \mathrm{z} 67) ; 8=$ (E)-ß-ocimene $(m / z$ 93); $9=$ linalool $(\mathrm{m} / \mathrm{z}$ 93); $10=$ methyl benzoate $(\mathrm{m} / \mathrm{z} 136) 11=1$-nonanol $(\mathrm{m} / \mathrm{z} 56) ; 12=$ methyl salicylate $(\mathrm{m} / \mathrm{z} 120) ; 13=$ ethyl salicylate $(\mathrm{m} / \mathrm{z} 120) ; 14=$ indole $(m / z 117) ; 15=(E, E)-\alpha$-farnesene $(\mathrm{m} / \mathrm{z} 93) ; 16=(E, E)-4,8$, 12-trimethyltrideca-1,3,7,11-tetraene (TMTT) $(m / z$ 69). Bars marked with $*$ indicate a treatment for a genotype significantly emitting more volatiles than its opposite uninfested or infested genotype. Bars marked with arrows represent compounds emitted in significantly different amounts by the two genotypes

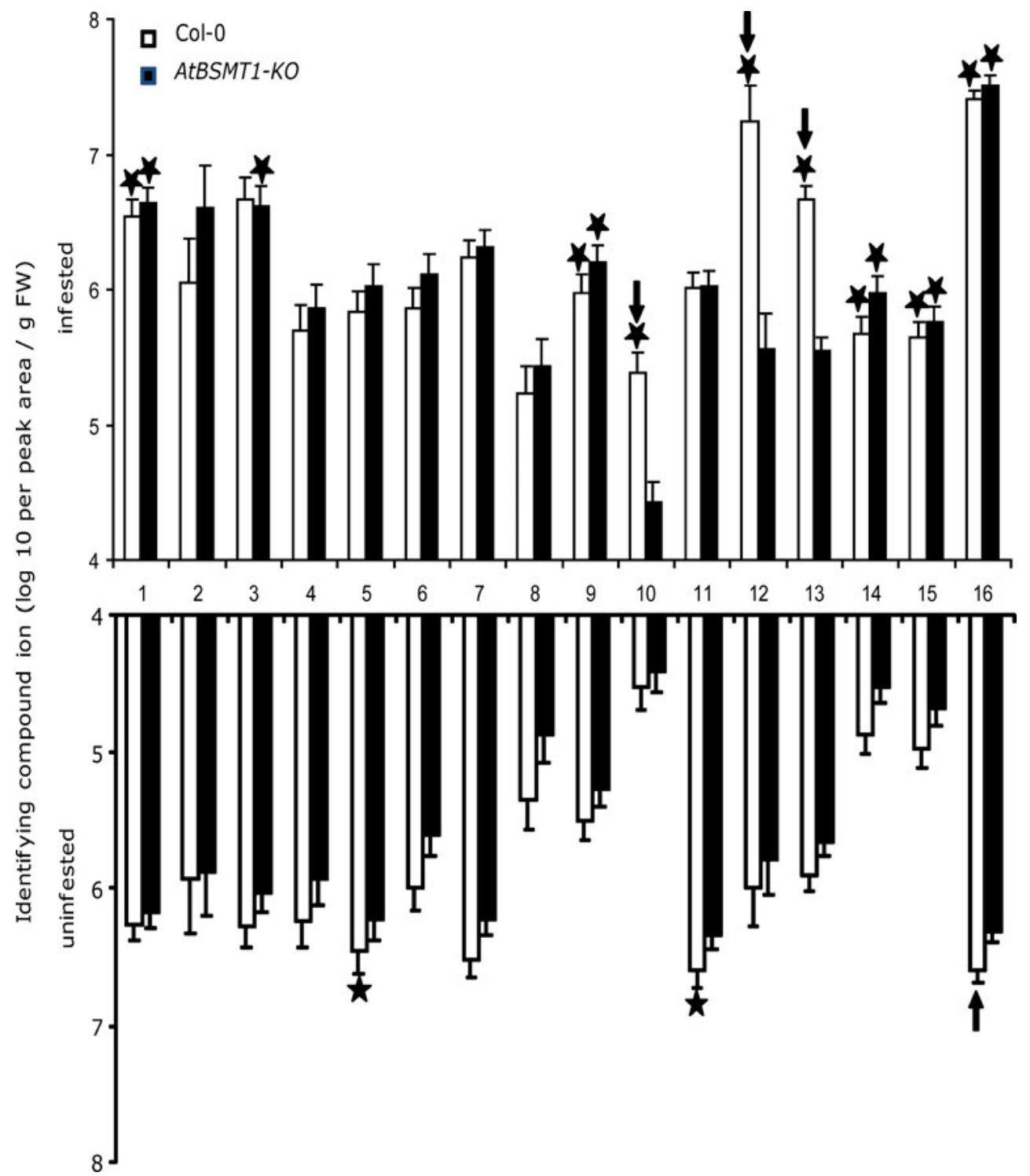

they preferred the latter. In contrast, no discrimination between odors from infested AtBSMT1-KO plants supplemented with 2 or $20 \mu \mathrm{g}$ MeSA vs. infested AtBSMT1-KO plants was observed.

The addition of MeSA negatively influenced the proportion of parasitoids that made a choice for one of the two odor sources. Analysis of the number of wasps that did not make a choice for one of the two odor sources, showed no effect of the offered genotype combination, but only an effect of the MeSA dose used (GLM: genotype combination $P=0.75$, MeSA dose $P=0.010, R^{2}=0.15$ ).

\section{Discussion}

Variation in the attraction of carnivorous arthropods to HIPVs often is ascribed to the relative contribution of attractive compounds within the complex herbivore- induced blend (Van Den Boom et al. 2004; D'Alessandro et al. 2006, 2009). Plant species can differ in the relative emission rates of individual attractive compounds in response to feeding by distinct herbivores. The commonly induced attractive compounds comprise green leaf volatiles (GLV), terpenoids, phenyl propanoids, and benzenoids. Several studies already have shown in planta the importance of some GLV and terpenoids in attracting carnivorous arthropods (Kappers et al. 2005; Schnee et al. 2006; Shiojiri et al. 2006). Yet, empirical evidence for the quantitative importance of single compounds in the total HIPV blend of infested plants is still rare (but see De Boer and Dicke 2004). The parasitoid D. semiclausum is attracted to the headspace of $P$. rapae-infested Col-0 plants (Loivamäki et al. 2008), in which MeSA is an induced volatile (Van Poecke et al. 2001). Here, we studied the effect of MeSA on preference for volatile blends in the parasitoid $D$. semiclausum. 


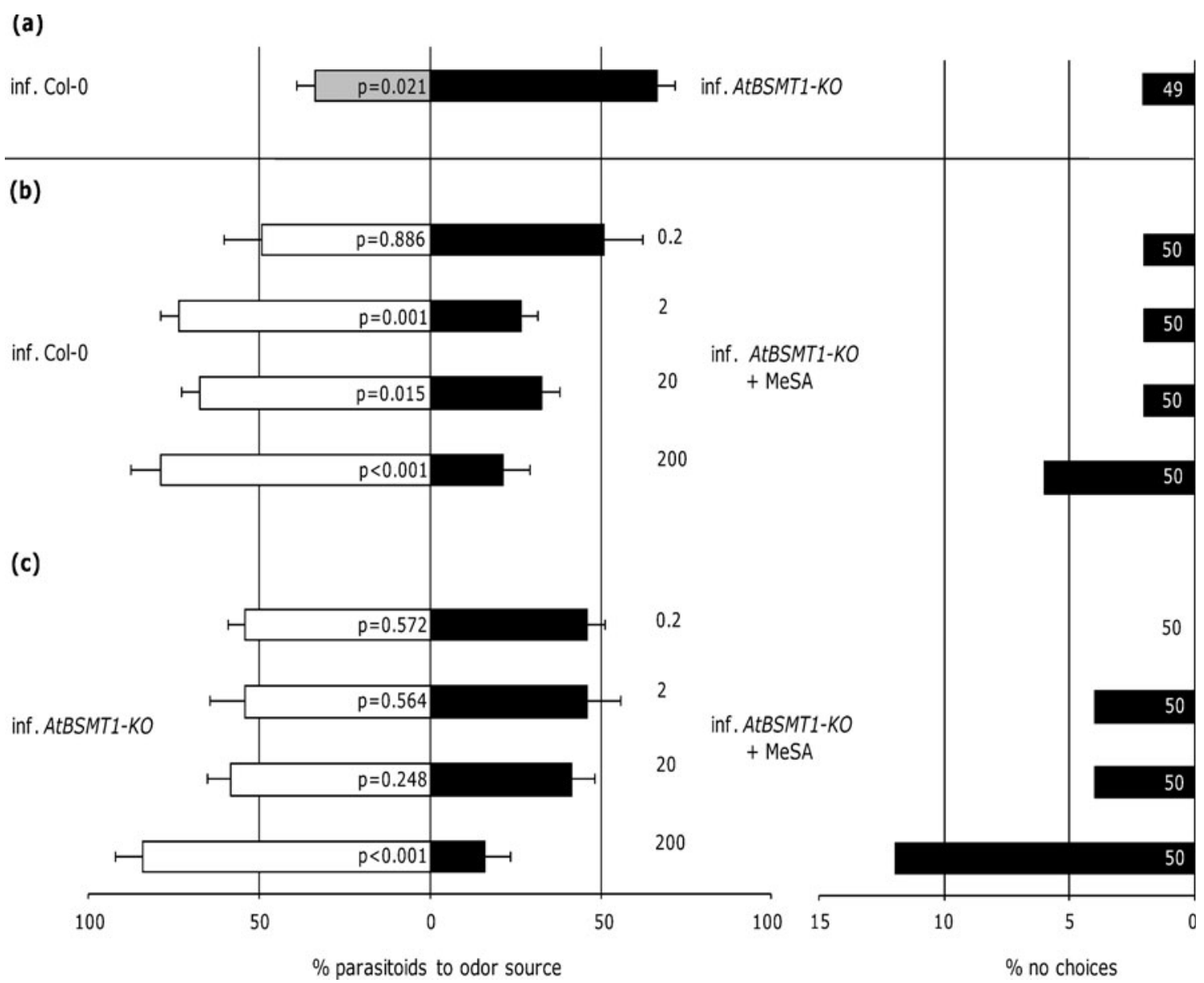

Fig. 3 Behavioral responses of naïve Diadegma semiclausum females to volatiles of two sets of Arabidopsis thaliana plants (Col-0 vs. AtBSMT1-KO), as assessed in the Y-tube olfactometer. All plants were infested (inf.) with 20 Pieris rapae caterpillars and in some cases the headspace was supplemented with synthetic methyl salicylate (Me$\mathrm{SA})$, added downwind from the plants. Added MeSA-doses $(\mu \mathrm{g})$ are indicated to the right of the bars in the left bar plot. Each bar represents the percentage of choices for each of the two odor sources as determined in five replicate experiments; on each replicate day 10 parasitoids were tested per odor source ( $X^{2}$ test, $P$ values). Error bars indicate SE. Generalized linear model analysis for experiments B and $\mathrm{C}$, demonstrated that MeSA dosage $(P<0.001)$ and not the offered genotype $(P=0.167)$ explained parasitoid behavior. Parasitoid MeSA dosage responses between two tested genotype combinations did not differ $(P=0.270)$. The right bar plots indicate the percentage of no choice in each experiment; total number of tested parasitoids are given in these bars
The newly-available Arabidopsis knock-out mutant for benzoic acid and salicylic acid carboxyl methyltransferase (AtBSMT1-KO) (Fig. 1, Fig. S1), allowed us to study in planta the ecological effects of an HIPV mixture lacking wild type levels of MeSA. Our data show clearly that caterpillar-infested AtBSMT1-KO plants attract more parasitoids than infested wild-type Col-0 plants (see Fig. 3a). Thus, although the total HIPV blend from wildtype plants strongly attracts $D$. semiclausum parasitoids (Loivamäki et al. 2008), a genotype that does not emit MeSA in response to caterpillar infestation is even more attractive to the parasitoids. These data indicate that MeSA does not contribute to the attraction of naïve $D$. semiclausum females but instead results in a reduced attractiveness.
This negative effect of MeSA on D. semiclausum attraction was not anticipated, as MeSA is commonly induced after herbivory in many plant species, e.g., in lima bean (Dicke et al. 1990), Brussels sprouts (Geervliet 1997; Bukovinszky et al. 2005), tomato (Ament et al. 2004), and Arabidopsis (Van Poecke et al. 2001; Chen et al. 2003). Moreover, the salicylic acid-deficient Arabidopsis mutant $N a h G$ is less attractive to Cotesia rubecula parasitoids upon infestation by $P$. rapae, which suggests an attractive role of MeSA for this parasitoid species (Van Poecke and Dicke 2002). Furthermore, synthetic MeSA has also been demonstrated to attract other carnivore species, such as predatory mites, lacewings, and mirid bugs (Dicke et al. 1990; De Boer and Dicke 2004; James and Price 2004). 
However, in our approach we tested the role of MeSA within the total HIPV-blend, by using AtBSMT1-KO plants with a hampered MeSA production. By additionally testing the response of the wasps to an HIPV blend from AtBSMT1-KO plants that we had supplemented with synthetic MeSA against HIPVs from Col-0 plants and AtBSMT1-KO plants, we demonstrated that MeSA acts as a repellent to naïve $D$. semiclausum. Moreover, the repellent effect of MeSA was dose-dependent, and this was reflected in the response level of the parasitoids, as fewer wasps move upwind to make a choice between odor sources with higher MeSA-doses. Additionally, the KOmutation did not affect the feeding rate of the caterpillars. Thus, the change in volatile emission and parasitoid attraction cannot be attributed to effects of the KOmutation. The data show that this compound plays a role in the observed repellent effects.

A few other studies also have recorded repellent effects of HIPV compounds to carnivores. For example, an HIPV fraction of maize, containing (Z)-3-hexen-1-ol acetate, linalool, and (3E)-4,8-dimethyl-1,3,7-nonatriene (DMNT) was found attractive compared to other tested fractions of the HIPV blend of maize to the parasitoid Cotesia marginiventris (Turlings and Fritzsche 1999). For the same plantherbivore system, naïve Microplitis rufiventris parasitoids preferred HIPV blends lacking the induced compound indole (D'Alessandro et al. 2006). Compounds that are repellent also may affect or override the attractiveness of other compounds in the headspace. Isoprenoids have, for example, been found to interfere with host-finding, as demonstrated for transgenic Arabidopsis plants emitting isoprene (Loivamäki et al. 2008). Therefore, to determine whether a hampered enzyme activity for SAMT and BAMT affected the headspace composition in other respects, we also analyzed the headspace of infested knock-out mutant and wild-type plants. We observed that the headspace of caterpillar-infested Col-0 and AtBSMT1-KO plants differed only in the emission of MeSA, MeBA, and EtSA. Yet, all other investigated HIPVs were emitted at similar rates by these infested plants (Fig. 2). The emission rates of the compounds whose rates were affected, were up to 60 times lower for AtBSMT1-KO plants compared to wild-type plants (Fig. 2). This agrees with the reported activity of the enzyme encoded by the BSMT1 gene (Chen et al. 2003). The very low emission of MeSA that remains may be ascribed to activity of another SABATH enzyme that shows low levels of activity towards SA and BA (Chen et al. 2003). Eliminating a functional BSMT1 gene also decreased the emission rate of EtSA. EtSA has been recorded in several plants (Hamilton-Kemp et al. 1988; Scutareanu et al. 1997; Deng et al. 2004) and is perceived by insect chemoreceptors (Ramachandran et al. 1990; Reinecke et al. 2002). Whether MeBA and EtSA also affect the attraction of $D$. semiclausum wasps remains to be investigated.

We investigated the role of MeSA by eliminating it from the total HIPV blend rather than by investigating its role as an isolated compound or as an addition to an artificial blend (see e.g., De Boer and Dicke 2004). We thus demonstrated that it has a repellent effect on the behavior of naïve $D$. semiclausum parasitoids. This finding provides another view on the fitness effects of individual components of herbivore-induced plant volatile blends that do not lure naïve carnivores but rather repel them. An alternative function of HIPV emitted compounds can be to repel herbivores (Dicke 1986; Bernasconi et al. 1998; Kessler and Baldwin 2001; Bruce et al. 2008; Piesik et al. 2008), which also has been demonstrated for MeSA (Hardie et al. 1994; James and Price 2004; Prinsloo et al. 2007; Ulland et al. 2008). MeSA may activate disease resistance and the expression of defense related genes in neighboring plants and in healthy tissue of infected plants (Shulaev et al. 1997). Therefore, the emission of MeSA also could benefit the plant through protecting it at herbivory-derived wounds against infectious pathogens, as has been reported for several GLVs (Brown et al. 1995; Shiojiri et al. 2006). In addition, evidence has accumulated for a plant-plant signaling role for MeSA (Ozawa et al. 2000; James and Price 2004), as well as indications for a priming effect of MeSA on plant defense (Turlings and Ton 2006).

A few studies indicate that parasitoids can learn to respond to individual compounds following a learning experience with an odor mixture (Meiners et al. 2003). This suggests that an innate response of D. semiclausum to avoid MeSA-emitting plants could potentially turn into attraction after a learning experience in the presence of a host.

In summary, our study provides evidence for an HIPV component that makes a plant less attractive to a carnivorous insect. This should be seen in the context of the different selection pressures on a plant's emission of volatiles in a multitrophic context. Understanding these selection pressures will provide insight into the role of induced volatiles in the biology of plants (Dicke and Baldwin 2010).

Acknowledgements The authors thank Leo Koopman, Frans van Aggelen, and André Gidding for culturing the insects, Iris Kappers for help with volatile analysis and fruitful discussions, Carola Ros for assistance with the behavioral assays, and Remco van Poecke for statistical assistance regarding volatile data. We thank Harro Bouwmeester for technical support at Plant Research International, and the Netherlands Organization for Scientific Research (NWO/ALW) for a VICI grant (865.03.002; T.A.L.S. and M.D.).

Open Access This article is distributed under the terms of the Creative Commons Attribution Noncommercial License which permits any noncommercial use, distribution, and reproduction in any medium, provided the original author(s) and source are credited. 


\section{References}

ADAMS, R. P. 1995. Identification of Essential Oil Components by Gas Chromatography/Mass Spectrometry. Allured Publishing Corporation, Illinois.

Ament, K., Kant, M. R., Sabelis, M. W., Haring, M. A., and SCHUURINK, R. C. 2004. Jasmonic acid is a key regulator of spider mite-induced volatile terpenoid and methyl salicylate emission in tomato. Plant Physiol. 135:2025-2037.

BAte, N. J., and RothsteIN, S. J. 1998. C-6-volatiles derived from the lipoxygenase pathway induce a subset of defense-related genes. Plant J. 16:561-569.

Benjamini, Y., and Hochberg, Y. 1995. Controlling the false discovery rate - A practical and powerful approach to multiple testing. J. Roy. Stat. Soc. Ser. B. (Stat. Method.) 57:289-300.

Bernasconi, M. L., Turlings, T. C. J., AmBrosetTi, L., Bassetti, P., and DoRN, S. 1998. Herbivore-induced emissions of maize volatiles repel the corn leaf aphid, Rhopalosiphum maidis. Entomol. Exp. Appl. 87:133-142.

Bleeker, M. A. K., Smid, H. M., Steidle, J. L. M., Kruidhof, H. M., VAN LoON, J. J. A., and Vet, L. E. M. 2006. Differences in memory dynamics between two closely related parasitoid wasp species. Anim. Behav. 71:1343-1350.

Brown, G. C., Prochaska, G. L., Hildebrand, D. F., Nordin, G. L., and JACKSON, D. M. 1995. Green leaf volatiles inhibit conidial germination of the entomopathogen Pandora neoaphidis (Entomopthorales: Entomophthoraceae). Environ. Entomol. 24:1637-1643.

Bruce, T. J. A., Matthes, M. C., Chamberlain, K., Woodcock, C. M., Mohib, A., Webster, B., Smart, L. E., Birkett, M. A., PicketT, J. A., and NAPIER, J. A. 2008. cis-Jasmone induces Arabidopsis genes that affect the chemical ecology of multitrophic interactions with aphids and their parasitoids. Proc. Natl. Acad. Sci. USA 105:4553-4558.

Bukovinszky, T., Gols, R., Posthumus, M. A., Vet, L. E. M., and VAN LENTEREN, J. C. 2005. Variation in plant volatiles and attraction of the parasitoid Diadegma semiclausum (Hellen). J. Chem. Ecol. 31:461-480.

Chen, F., D'auria, J. C., Tholl, D., Ross, J. R., Gershenzon, J., NoEl, J. P., and PICHERSKY, E. 2003. An Arabidopsis thaliana gene for methylsalicylate biosynthesis, identified by a biochemical genomics approach, has a role in defense. Plant J. 36:577-588.

D'Alessandro, M., and Turlings, T. C. J. 2006. Advances and challenges in the identification of volatiles that mediate interactions among plants and arthropods. Analyst 131:24-32.

D’alessandro, M., Held, M., Triponez, Y., and Turlings, T. C. J. 2006. The role of indole and other shikimic acid derived maize volatiles in the attraction of two parasitic wasps. J. Chem. Ecol. 32:2733-2748.

D'alessandro, M., Brunner, V., Von Merey, G., and Turlings, T. C. 2009. Strong Attraction of the Parasitoid Cotesia marginiventris Towards Minor Volatile Compounds of Maize. J. Chem. Ecol. 35:999-1008.

DE BoER, J. G., and Dicke, M. 2004. The role of methyl salicylate in prey searching behaviour of the predatory mite Phytoseiulus persimilis. J. Chem. Ecol. 30:255-271.

De Boer, J. G., Posthumus, M. A., and Dicke, M. 2004. Identification of volatiles that are used in discrimination between plants infested with prey or nonprey herbivores by a predatory mite. J. Chem. Ecol. 30:2215-2230.

Deng, C. H., Zhang, X. M., ZhU, W. M., and QIAN, J. 2004. Gas chromatography-mass spectrometry with solid-phase microextraction method for determination of methyl salicylate and other volatile compounds in leaves of Lycopersicon esculentum. Anal. Bioanal. Chem. 378:518-522.
De Vos, M., Van Oosten, V. R., Van Poecke, R. M. P., Van Pelt, J. A., Pozo, M. J., Mueller, M. J., Buchala, A. J., MetrauX, J. P., VAN LOON, L. C., DickE, M. et al. 2005. Signal signature and transcriptome changes of Arabidopsis during pathogen and insect attack. Mol. Plant-Microbe Interact. 18:923-937.

Dicke, M. 1986. Volatile spider-mite pheromone and host-plant kairomone, involved in spaced-out gregariousness in the spider mite Tetranychus urticae. Physiol. Entomol. 11:251-262.

DiCKE, M. 1999a. Are herbivore-induced plant volatiles reliable indicators of herbivore identity to foraging carnivorous arthropods? Entomol. Exp. Appl. 91:131-142.

DiCKE, M. 1999b. Evolution of induced indirect defense of plants. pp. 62-88, in R. Tollrian and C. J. Harvell (eds.). The Ecology And Evolution Of Inducible Defenses: Princeton University Press, Princeton, New Jersey.

DickE, M., and BALDwIN, I. T. 2010. The evolutionary context for herbivore-induced plant volatiles: beyond the cry for help Trends Plant Sci. 15:167-175.

Dicke, M., and VAN PoECKE, R. M. P. 2002. Signaling in plant-insect interactions: signal transduction in direct and indirect plant defence, pp. 289-316, in D. Scheel and C. Wasternack (eds.). Plant Signal Transduction. Oxford University Press, Oxford, UK.

Dicke, M., Van Beek, T. A., Posthumus, M. A., Ben Dom, N., VAN BoKhoven, H., and De Groot, A. E. 1990. Isolation and identification of volatile kairomone that affects acarine predator - prey interactions. Involvement of host plant in its production. $J$. Chem. Ecol. 16:381-396.

Dicke, M., Gols, R., Ludeking, D., and Posthumus, M. A. 1999. Jasmonic acid and herbivory differentially induce carnivoreattracting plant volatiles in lima bean plants. J. Chem. Ecol. 25:1907-1922.

Du, Y. J., Poppy, G. M., Powell, W., Pickett, J. A., Wadhams, L. J., and WoODCOCK, C. M. 1998. Identification of semiochemicals released during aphid feeding that attract parasitoid Aphidius ervi. J. Chem. Ecol. 24:1355-1368.

Dudareva, N., Negre, F., Nagegowda, D. A., and Orlova, I. 2006. Plant volatiles: Recent advances and future perspectives. Crit. Rev. Plant Sci. 25:417-440.

Fatouros, N. E., VAn LoOn, J. J. A., HordiJK, K. A., Smid, H. M., and DiCKE, M. 2005. Herbivore-induced plant volatiles mediate in-flight host discrimination by parasitoids. J. Chem. Ecol. 31:2033-2047.

GeERVLIET, J. B. F. 1997. Infochemical use by insect parasitoids in a tritrophic context: comparison of a generalist and a specialist $\mathrm{PhD}$ dissertation.

Gershenzon, J., and Dudareva, N. 2007. The function of terpene natural products in the natural world. Nat. Chem. Biol. 3:408-414.

Halitschke, R., Stenberg, J. A., Kessler, D., Kessler, A., and BALDWIN, I. T. 2008. Shared signals-'alarm calls' from plants increase apparency to herbivores and their enemies in nature. Ecol. Lett. 11:24-34.

Hamilton-Kemp, T. R., Andersen, R. A., Rodriguez, J. G., Loughrin, J. H., and PAtTerson, C. G. 1988. Strawberry foliage headspace vapor components at periods of susceptibility and resistance to Tetranychus urticae Koch. J. Chem. Ecol. 14:789-796.

Hardie, J., IsAacs, R., Pickett, J. A., Wadhams, L. J., and Woodcock, C. M. 1994. Methyl salicylate and (-)-(1R,5S)myrtenal are plant-derived repellents for black bean aphid, Aphis fabae Scop. (Homoptera: Aphididae). J. Chem. Ecol. 20:28472855.

HeIDEL, A. J., and BALDwIN, I. T. 2004. Microarray analysis of salicylic acid- and jasmonic acid-signalling in responses of Nicotiana attenuata to attack by insects from multiple feeding guilds. Plant Cell Environ. 27:1362-1373. 
ISHIWARI, H., SUZUKI, T., and MAEDA, T. 2007. Essential compounds in herbivore-induced plant volatiles that attract the predatory mite Neoseiulus womersleyi. J. Chem. Ecol. 33:1670-1681.

JAMES, D. G., and PRICE, T. S. 2004. Field-testing of methyl salicylate for recruitment and retention of beneficial insects in grapes and hops. J. Chem. Ecol. 30:1613-1628.

KAISER, L., and CARDE, R. T. 1992. In-flight orientation to volatiles from the plant-host complex in Cotesia rubecula (Hym.: Braconidae): increased sensitivity through olfactory experience. Physiol. Entomol. 17:62-67.

Kappers, I. F., Aharoni, A., VAn Herpen, T., LuCKerhoff, L. L. P., Dicke, M., and BouWMEESTER, H. J. 2005. Genetic engineering of terpenoid metabolism attracts, bodyguards to Arabidopsis. Science 309:2070-2072.

Kapteyn, J., Qualley, A. V., Xie, Z. Z., Fridman, E., Dudareva, N., and GANG, D. R. 2007. Evolution of cinnamate/p-coumarate carboxyl Methyltransferases and their role in the biosynthesis of methylcinnamate. Plant Cell 19:3212-3229.

Kessler, A., and BALDWIN, I. T. 2001. Defensive function of herbivore-induced plant volatile emissions in nature. Science 291:2141-2144.

Kessler, A., and Baldwin, I. T. 2002. Plant responses to insect herbivory: The emerging molecular analysis. Annu. Rev. Plant Biol. 53:299-328.

Kost, C., and HeIL, M. 2008. The defensive role of volatile emission and extrafloral nectar secretion for lima bean in nature. J. Chem. Ecol. 34:2-13.

Leitner, M., Boland, W., and Mithofer, A. 2005. Direct and indirect defences induced by piercing-sucking and chewing herbivores in Medicago truncatula. New Phytol. 167:597-606.

LOIVAMÄKI, M., MUMM, R., DicKE, M., and SCHNITZLER, J.-P. 2008. Isoprene interferes with the attraction of bodyguards by herbaceous plants. Proc. Natl. Acad. Sci. USA 105:17430 17435 .

MeInERS, T., WACKers, F., and LeWIS, W. J. 2003. Associative learning of complex odours in parasitoid host location. Chem. Senses 28:231-236

Murfitt, L. M., Kolosova, N., Mann, C. J., and Dudareva, N. 2000. Purification and characterization of S-adenosyl-L-methionine: Benzoic acid carboxyl methyltransferase, the enzyme responsible for biosynthesis of the volatile ester methyl benzoate in flowers of Antirrhinum majus. Archives of Biochemistry and Biophysics 382:145-151.

Ozawa, R., Arimura, G., Takabayashi, J., Shimoda, T., and NISHIOKA, T. 2000. Involvement of jasmonate- and salicylaterelated signaling pathways for the production of specific herbivore-induced volatiles in plants. Plant Cell Physiol. 41:391-398.

PARÉ, P. W., and TUMLINSON, J. H. 1997. De novo biosynthesis of volatiles induced by insect herbivory in cotton plants. Plant Physiol. 114:1161-1167.

Piesik, D., Weaver, D. K., Runyon, J. B., Buteler, M., PeCK, G. E., and MorriLl, W. L. 2008. Behavioural responses of wheat stem sawflies to wheat volatiles. Agric. For. Entomol. 10:245253

Poelman, E. H., Oduor, A. M. O., Broekgaarden, C., HordiJK, C. A., Jansen, J. J., VAN LoOn, J. J. A., VAN DAM, N. M., Vet, L. E. M., and DickE, M. 2009. Field parasitism rates of caterpillars on Brassica oleracea plants are reliably predicted by differential attraction of Cotesia parasitoids. Funct. Ecol. 23:951962.

Prinsloo, G., Ninkovic, V., Van Der Linde, T. C., VAn Der Westhuizen, A. J., Pettersson, J., and Glinwood, R. 2007. Test of semiochemicals and a resistant wheat variety for Russian wheat aphid management in South Africa. J. Appl. Entomol. 131:637-644.
RAMACHANDRAN, R., Khan, Z. R., CABAllero, P., and Juliano, B. O. 1990. Olfactory sensitivity of two sympatric species of rice leaf folders (Lepidoptera: Pyralidae) to plant volatiles. J. Chem. Ecol. 16:2647-2666

Reinecke, A., Ruther, J., Tolasch, T., Francke, W., and Hilker, M. 2002. Alcoholism in cockchafers: orientation of male Melolontha melolontha towards green leaf alcohols. Naturwissenschaften 89:265-269.

Schmidt, D. D., Voelckel, C., Hartl, M., Schmidt, S., and BALDWIN, I. T. 2005. Specificity in ecological interactions. Attack from the same lepidopteran herbivore results in speciesspecific transcriptional responses in two solanaceous host plants. Plant Physiol. 138:1763-1773.

Schnee, C., Kollner, T. G., Held, M., Turlings, T. C. J., GERShEnZON, J., and DEgEnhARDT, J. 2006. The products of a single maize sesquiterpene synthase form a volatile defense signal that attracts natural enemies of maize herbivores. Proc. Natl. Acad. Sci. USA 103:1129-1134.

SCHROEDER, R., and HILKER, M. 2008. The relevance of background odor in resource location by insects: A behavioral approach. Bioscience 58:308-316.

Scutareanu, P., Drukker, B., Bruin, J., Posthumus, M. A., and SABELIS, M. W. 1997. Volatiles from Psylla-infested pear trees and their possible involvement in attraction of anthocorid predators. J. Chem. Ecol. 23:2241-2260.

Seo, H. S., Song, J. T., Cheong, J. J., LeE, Y. H., Lee, Y. W. Hwang, I., LeE, J. S., and ChOI, Y. D. 2001. Jasmonic acid carboxyl methyltransferase: A key enzyme for jasmonateregulated plant responses. Proc. Natl. Acad. Sci. USA 98:47884793.

SHIMOdA, T., OZAWA, R., SANO, K., YANO, E., and TAKABAYASHI, J. 2005. The involvement of volatile infochemicals from spider mites and from food-plants in prey location of the generalist predatory mite Neoseiulus californicus. J. Chem. Ecol. 31:20192032 .

Shiojiri, K., Kishimoto, K., OzaWA, R., Kugimiya, S., Urashimo, S., Arimura, G., Horiuchi, J., Nishioka, T., Matsui, K., and TAKABAYASHI, J. 2006. Changing green leaf volatile biosynthesis in plants: An approach for improving plant resistance against both herbivores and pathogens. Proc. Natl. Acad. Sci. USA 103:16672-16676.

Shulaev, V., Silverman, P., and Raskin, I. 1997. Airborne signalling by methyl salicylate in plant pathogen resistance. Nature 385:718-721.

SMID, H. M. 2006. Variation in learning of herbivory-induced plant odours by parasitic wasps, pp. 88-103, in M. Dicke and W. Takken (eds.). Chemical Ecology: From Gene to Ecosystem. Springer, Dordrecht.

Snoeren, T. A. L., De Jong, P. W., and Dicke, M. 2007. Ecogenomic approach to the role of herbivore-induced plant volatiles in community ecology. J. Ecol. 95:17-26.

Snoeren, T. A. L., VAn Poecke, R. M. P., and Dicke, M. 2009. Multidisciplinary approach to unravelling the relative contribution of different Oxylipins in Indirect Defense of Arabidopsis thaliana. J. Chem. Ecol. 35:1031.

TurLINGS, T. C. J., and FriTZSCHE, M. E. 1999. Attraction of parasitic wasps by caterpillar-damaged plants, pp. 21-32, in D. J. CHADWICK and J. GOODE (eds.). Insect-Plant Interactions and Induced Plant Defence (Novartis Foundation Symposium 223). Wiley, Chicester.

TURLINGS, T. C. J., and TON, J. 2006. Exploiting scents of distress: the prospect of manipulating herbivore-induced plant odours to enhance the control of agricultural pests. Curr. Opin. Plant Biol. 9:421-427.

Turlings, T. C. J., Tumlinson, J. H., Heath, R. R., ProveauX, A. T., and DoOlitTle, R. E. 1991. Isolation and identification of 
allelochemicals that attract the larval parasitoid, Cotesia marginiventris (Cresson), to the microhabitat of one of its hosts. J. Chem. Ecol. 17:2235-2251.

Ulland, S., Ian, E., Mozuraitis, R., Borg-Karlson, A. K., Meadow, R., and Mustaparta, H. 2008. Methyl salicylate, identified as primary odorant of a specific receptor neuron type, inhibits oviposition by the moth Mamestra brassicae L. (Lepidoptera, Noctuidae). Chem. Senses 33:35-46.

Van Den Boom, C. E. M., Van Beek, T. A., Posthumus, M. A., De Groot, A., and DiCKE, M. 2004. Qualitative and quantitative variation among volatile profiles induced by Tetranychus urticae feeding on plants from various families. J. Chem. Ecol. 30:69-89.

VAN POECKE, R. M. P., and DiCKE, M. 2002. Induced parasitoid attraction by Arabidopsis thaliana: involvement of the octadecanoid and the salicylic acid pathway. J. Exp. Bot. 53:1793-1799.

Van Poecke, R. M. P., Posthumus, M. A., and Dicke, M. 2001. Herbivore-induced volatile production by Arabidopsis thaliana leads to attraction of the parasitoid Cotesia rubecula: Chemical, behavioral, and gene-expression analysis. J. Chem. Ecol. 27:1911-1928.
Vet, L. E. M., and DicKE, M. 1992. Ecology of infochemical use by natural enemies in a tritrophic context. Annu. Rev. Entomol. No. 37:141-172.

WÄCKers, F. L., Zuber, D., Wunderlin, R., and Keller, F. 2001. The effect of herbivory on temporal and spatial dynamics of foliar nectar production in cotton and castor. Ann. Bot. 87:365370.

WALLING, L. L. 2000. The myriad plant responses to herbivores. $J$. Plant Growth Regul. 19:195-216.

WitTstock, U., and Gershenzon, J. 2002. Constitutive plant toxins and their role in defense against herbivores and pathogens. Curr. Opin. Plant Biol. 5:300-307.

ZHU, J. W., and PARK, K. C. 2005. Methyl salicylate, a soybean aphidinduced plant volatile attractive to the predator Coccinella septempunctata. J. Chem. Ecol. 31:1733-1746.

Zubieta, C., Ross, J. R., Koscheski, P., YANG, Y., Pichersky, E., and NOEL, J. P. 2003. Structural basis for substrate recognition in the salicylic acid carboxyl methyltransferase family. Plant Cell 15:1704-1716. 